University of Wollongong

Research Online

Faculty of Engineering and Information

Faculty of Engineering and Information

Sciences - Papers: Part A

Sciences

$1-1-2014$

\title{
An approach for online assessment of rooftop solar PV impacts on low- voltage distribution networks
}

Md Jan-E- Alam

University of Wollongong, mjea982@uowmail.edu.au

Kashem Muttaqi

University of Wollongong, kashem@uow.edu.au

Darmawan Sutanto

University of Wollongong, soetanto@uow.edu.au

Follow this and additional works at: https://ro.uow.edu.au/eispapers

Part of the Engineering Commons, and the Science and Technology Studies Commons

Research Online is the open access institutional repository for the University of Wollongong. For further information contact the UOW Library: research-pubs@uow.edu.au 


\title{
An approach for online assessment of rooftop solar PV impacts on low-voltage distribution networks
}

\begin{abstract}
Assumption-based offline analysis tools may not be able to provide sufficient and accurate information for the corrective decision making to mitigate solar photovoltaic (PV) impacts in the future distribution grids. This is mainly due to the increasing penetration level of intermittent power generation resources and also the fluctuating behavior of consumer demand. Online assessment tools can assist to manage PV impacts and aid to mitigate those on a real-time basis. This paper proposes an approach for online assessment of rooftop PV impacts on low-voltage (LV) networks using real-time network data. A variablewidth sliding window will be used to provide the analysis an outcome-based online data. The width of the sliding window can be varied according to user input so that the changes in network behavior caused by PV integration can be investigated conveniently. Several numerical indices are proposed in this paper to assess solar PV impacts on the LV networks. This approach also uses the online data to develop real-time distribution network models for a dynamic "what-if" analysis. The usefulness of the proposed online assessment approach is verified using an Australian LV distribution feeder.
\end{abstract}

\section{Keywords}

solar, pv, rooftop, voltage, assessment, line, approach, distribution, networks, low, impacts

\author{
Disciplines \\ Engineering | Science and Technology Studies
}

\section{Publication Details}

M. J. E. Alam, K. M. Muttaqi \& D. Sutanto, "An approach for online assessment of rooftop solar PV impacts on low-voltage distribution networks," IEEE Transactions on Sustainable Energy, vol. 5, (2) pp. 663-672, 2014. 


\title{
An Approach for On-line Assessment of Rooftop Solar PV Impacts on Low Voltage Distribution Networks
}

\author{
M. J. E. Alam, Student Member, IEEE, K. M. Muttaqi, Senior Member, IEEE, and D. Sutanto, Senior \\ Member, IEEE
}

\begin{abstract}
Assumption based off-line analysis tools may not be able to provide sufficient and accurate information for corrective decision-making to mitigate solar PV impacts in the future distribution grids. This is mainly due to the increasing penetration level of intermittent power generation resources and also the fluctuating behavior of consumer demand. On-line assessment tools can assist to manage PV impacts and aid to mitigate those on real time basis. This paper proposes an approach for on-line assessment of rooftop PV impacts on low voltage (LV) networks using real-time network data. A variablewidth sliding window will be used to provide the analysis outcomes based on-line data. The width of the sliding window can be varied according to user input so that the changes in network behavior caused by PV integration can be investigated conveniently. Several numerical indices are proposed in this paper to assess solar PV impacts on the LV networks. This approach also uses the on-line data to develop real time distribution network models for a dynamic "what-if" analysis. The usefulness of the proposed on-line assessment approach is verified using an Australian LV distribution feeder.
\end{abstract}

Index Terms-Solar PV impact, on-line assessment, sliding window, LV distribution networks, real-time network data.

\section{INTRODUCTION}

The penetration of rooftop solar PV resources is increasing in the low voltage (LV) distribution networks day by day. With the capability to serve loads at the connection point, PV resources have the potential to change the normal network behavior. While the integration of solar PV can provide different system support benefits [1], a high penetration of PV on the other hand may impact the network in several ways [2], such as voltage rise, reverse power flow, and increased energy loss [3]. In many countries around the world, the integration of PV resources at a high penetration level is a comparatively new issue. Many utilities are yet to fully understand the consequences of the cascaded impacts in a real time operation. Network operators therefore deploy analytical tools to assess the potential impacts of PV resources on the distribution networks. In general, off-line impact

This work is supported by the Australian Research Council (ARC) and Essential Energy Linkage Grant, LP100100618.

M. J. E. Alam, K. M. Muttaqi and D. Sutanto is with Australian Powe Quality and Reliability Center, School of Electrical Computer and Telecommunications Engineering, University of Wollongong, NSW 2522, Australia (e-mail: mjea982@uowmail.edu.au, kashem@uow.edu.au, soetanto@uow.edu.au). analysis is performed during the processing of a prospective PV connection request using assumption based synthetic data, and/or, historical data collected and archived through monitoring devices installed in the networks. While the impact studies during acceptance of a PV connection request can provide a preliminary estimation of $\mathrm{PV}$ impacts, the real scenario in the presence of varying load and network conditions are still unknown. Also, new PV connections are being accepted regularly leading to change of the prevailing conditions during the acceptance of the connection requests. Therefore, an off-line investigation performed before may not be adequate for the present scenario. Similarly, off-line analysis using the historical data may not be sufficient for taking appropriate mitigation actions due to the changes in network condition with time, such as addition of loads and network equipments, and installation of new generation resources.

With the increasing level of solar PV penetration in distribution networks, advanced tools would be needed that are capable of online impact assessment so that sufficient information on PV impacts are available from the most recent network condition with an acceptable accuracy. This will require a collection of real time system operation data and the ability to perform the impact analysis on-line so that network operators can take immediate decisions and implement necessary actions at the time of occurrence of any detrimental impact.

Extensive research has been carried out on modeling and analysis of distribution networks with Distributed Generation (DG) resources based on a general off-line approach; a review is provided in [4]. Several numerical indices have been developed in [5] for the analysis of network impacts of DG resources to investigate the best locations for DG units in the network using a steady-state analysis approach. This type of approach is useful for off-line analysis, typically performed in planning oriented studies for DG integration. A general approach to quantify the benefits of DG integration has been discussed in [6] using a parametric study where the variations of different DG impact indices have been observed with the variations of DG ratings and DG power factor. Again, the analytical approach used is more suitable for an off-line type analysis using previously archived data. In contrast, the motivation of the research in this paper is to assess the PV impacts on-line using real-time data so that a more accurate 
analysis of the impacts could be done under the varying load and network conditions. This would also be helpful to take a more effective decision (as the impact estimation is more accurate) on the spot for immediate mitigation by available means.

This paper proposes a variable-width sliding window based on-line assessment approach for solar PV impacts on LV distribution networks. The proposed approach will use the data available from numerous smart meters installed in the future smart grid environment to assess solar PV impacts online. Using the variable-width sliding window allows the width of the assessment window to be varied depending on the situation. For example, when the PV output is at its peak level, the width of the assessment window can be reduced to obtain a closer view of the situation, because the threat of the voltage limit violation could be high at this time. On the other hand, when the PV output is consistently decreasing or unavailable, for example during late afternoon or at night, the width of the sliding window can be increased to avoid unnecessary monitoring activities. Different numerical indices will be defined based on key network parameters that are influenced by the PV integration. Real time network models will be compiled using the most recent network information to investigate the impact of PV penetration by incorporating any desired changes in the PV output or network parameters. The proposed approach will be able to perform a "what-if" analysis using a time-varying base case scenario to advise the user how a given operating network would behave in a potential PV generation scenario. The information obtained using the proposed approach would be beneficial (in terms of a more accurate impact analysis) to take mitigation actions against adverse impacts of high PV penetration, including fluctuations caused by sudden variations in PV output. The applicability of the approach will be tested using a practical LV distribution feeder in Australia.

\section{The Proposed ON-Line Assessment ApPrOACH}

Offline analysis of rooftop solar PV impacts on LV networks is typically performed using assumption based data due to unavailability of detailed operational data on the LV networks. Scenario analysis is performed using load flow solution with the load and PV generation data modified to fulfill the analysis objectives, such as the analysis of worstcase scenario. The proposed on-line approach on the other hand, will perform the impact investigations using real time data collected by smart meters installed at different locations in the network, and the usage of assumption will be kept as low as possible. Functionalities of the proposed approach are described below.

\section{A. Architecture of the Proposed On-line Approach}

The proposed approach will consist of on-line data input functionalities, as shown in Fig. 1, that will receive real time data related to distribution networks and also the weather information that is related to PV power output. The approach will also have the ability to update the network topology using the most recent switching and outage information. Combining the available on-line network data and topological information, the proposed approach would be able to make use of real time models of distribution networks. Solar PV impacts on networks will be assessed by the approach using several numerical indices based on network variables that are mostly affected by solar PV, such as, bus voltage, line power flow, and feeder power loss. The proposed approach and indices will be highly useful to assess the impact of an increasingly high penetration of solar PV.

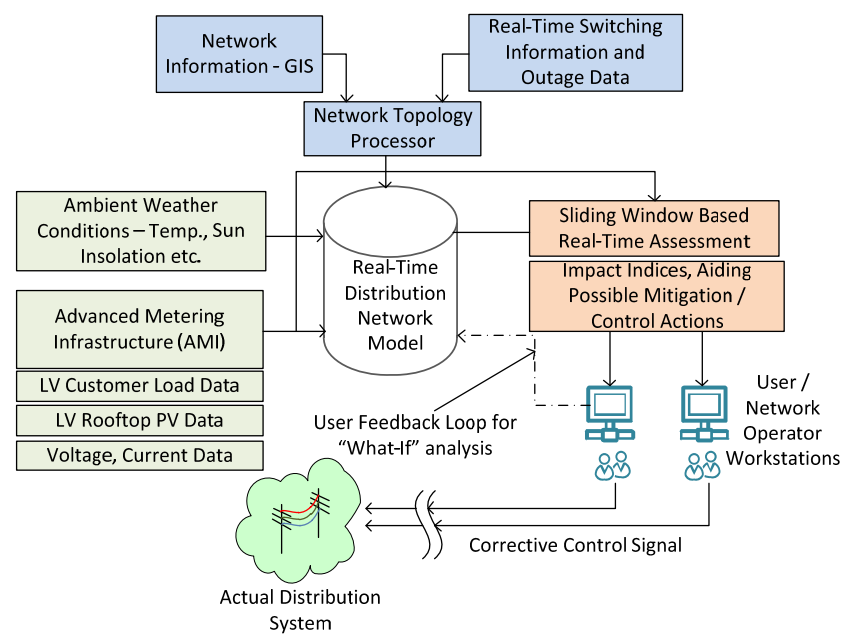

Fig. 1. The architecture of the proposed online assessment approach

\section{B. Collection and Processing of Real Time Data with AMI}

Real time system operation data will collected from numerous smart meters and monitoring devices anticipated in the future distribution networks using an Advanced Metering Infrastructure (AMI) [7]. Resolution of the data will depend on the technical specifications of the AMI devices. If required, data pre-processing techniques [8] will be applied to improve the quality of the measured data.

\section{Sliding Window Based Assessment}

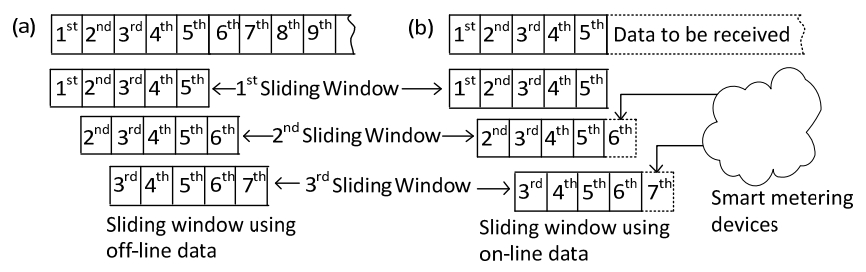

Fig. 2. Illustration of sliding window; (a) using on-line data; (b) using off-line data.

Sliding windows are normally used for extracting subsequences from stored (i.e. offline) time-series data. Fig. 2 shows an illustrative example of how this can be applied for extraction of time series sub-sequences from on-line data. An off-line time series data of an arbitrary length is considered in Fig. 2(a), where up to the $9^{\text {th }}$ data point is shown. In general, the $i^{\text {th }}$ sub-sequence, $\mathbf{S}_{i}$, of length $W$ from an $N$-dimensional time series data set, $\mathbf{D}$, can be extracted using (1).

$$
\mathbf{S}_{i}=\mathbf{D}(i: m)
$$

where, $m=(i+W-1)$ and, $i=1,2, \ldots, N-(W-1)$.

Following this and using a sliding window of length 5 , for example, the $1^{\text {st }}$ sub-sequence is a vector of $1^{\text {st }}$ to $5^{\text {th }}$ data 
points; the $2^{\text {nd }}$ one is a vector of $2^{\text {nd }}$ to $6^{\text {th }}$, and so on. It is observed that the $m^{\text {th }}$ data point is always available as long as archived off-line data is considered. This is, however, not the case with on-line data, where the $m^{\text {th }}$ data has to be obtained from real time measurements. The example in Fig. 2(b) shows that the $2^{\text {nd }}$ sub-sequence is formed by combining the $2^{\text {nd }}$ to $5^{\text {th }}$ previously received data points with the $6^{\text {th }}$ recently received data; similarly, the $3^{\text {rd }}$ sub-sequence is obtained from the $3^{\text {rd }}$ to $6^{\text {th }}$ previously received data with the recently received $7^{\text {th }}$ data, and so on. These sub-sequences of time series data are used as the sliding windows for the proposed on-line approach.
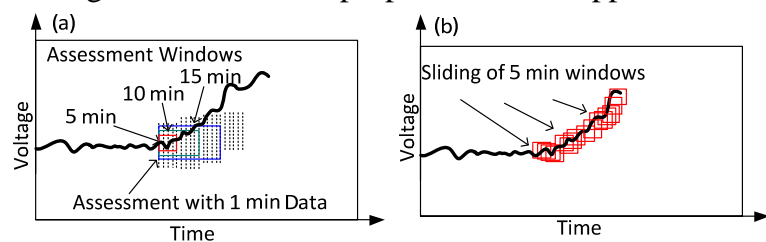

Fig. 3. Sliding window based PV impact assessment: (a) 5, 10 and 15-min assessment windows (b) sliding of 5-min windows.

Solar PV impacts in terms of changes in key network parameters will be assessed based on 1-min data logged by smart meters. The 1-min assessment results will then be used to develop a variable sliding window based assessment report. This concept of sliding window is described below using a hypothetical voltage profile time series data shown in Fig. 3 with 5-min, 10-min and 15-min assessment windows. The width of the sliding window can be defined by the users to obtain the assessment outcomes in a customized way.

The voltage profile in Fig. 3 shows a rising trend due to the integration of solar PV resources. Consecutive 5 samples of 1min interval data are used for building the 5-min assessment window; similarly 10 instances of 1-min data are used for 10min assessment window and so on. Each of the windows progresses (slide) by 1-min to provide a sliding window based assessment. This is shown in Fig. 3(b) for the 5-min window only; other widths will follow the similar pattern. The first window provides the assessment results for 5 time instances, from instance 1 to 5 ; the second window gives the results for instance 2 to 6 and so on.

The width, $W$, of a sliding window can be any value between 1 and the number of data points already received, and it is mainly a user defined parameter. In terms of time period, the narrowest width will depend on the resolution of the online data available from the metering systems. For example, if the data are measured in second range, the width of the window can be varied from seconds to minutes to up to hours. On the other hand, if the data is measured in minutes (e.g. 1 minute resolution) then the narrowest width would be in terms of minutes. Resolution of the on-line data is also crucial for assessment of PV output fluctuations and associated impacts. If the time period between two consecutive measurements is higher than the time duration of the fluctuation event, then it will not be visible in the on-line data and the impacts cannot be assessed from such low resolution of the online data. Therefore, the higher the resolution of the online data, the wider would be the choice of the width of an assessment window.

Although for a given resolution of the online data, the width of the assessment window is mainly a user defined parameter, still a suggestion can made on the selection of an appropriate width using the previous measurements of the solar irradiance level. Logically, the width needs to become narrow with the increase of severity of the PV impacts, which depend on the solar irradiance level for a given set of network conditions. Therefore, suggestion can be made to decrease the width (in terms of time) with the increase of the irradiance level and vice versa.

The advantage of a variable-width sliding window is its adjustability to provide the assessment outcomes in a more customizable way that suits the assessment situation better. For example, in a mostly cloudy day, the impact of PV may not be significantly visible and the user can choose to use a wide assessment window, such as $30 \mathrm{~min}$, or $60 \mathrm{~min}$. On the other hand, in a clear day with the PV units generating at their maximum capacity at noon, when the load demand is usually low, a narrow assessment window, e.g. $10 \mathrm{~min}$, or, $5 \mathrm{~min}$, would be desirable to obtain a closer and tighter view of the PV impacts, such as, a possible violation of the overvoltage limit.

\section{Real Time Model of Distribution Networks}

A traditional model of a distribution network is an assembly of network components in terms of their admittances combined according to the network topology and the loads imposed at respective buses. With the integration of solar PV resources, the network model will also include PV power injections at buses where PV units are connected. An off-line model of a distribution network can be constructed from the topological information obtained from Geographic Information System (GIS) maps, the design data of the network, planned or historical load demand data, and nominal or historical output data of PV units. On the other hand, a real time model of a distribution network would need the most recent information and data on the network. A network topology processor will be used for on-line updating of the network admittance model by incorporating any change in the designed topology due to switching and/or outage of network components. Load demand and PV output data will be updated using the on-line data obtained from AMI. The weather data related to PV output will also be available for different types of scenario analysis, or case studies in changed weather conditions.

\section{E. Fast and Accurate Network Analysis Tool}

While solar PV impacts can be investigated based on the online measurements of voltages and power flows, simulation oriented analysis using the real time load and PV generation data can be performed to make an online evaluation of impacts from measured data and compare the results with the actual impacts to check the validity of the real time network models. However, a more important application of the simulation would be to perform "what-if" and "worst-case" scenario analysis by modifying the metered PV generation data. To perform the simulation oriented analysis on-line, the proposed approach needs to utilize an algorithm that is fast but accurate enough so that correct assessment outcomes can be made in 
time. For example, an on-line assessment tool designed for performing the analysis with 1-min resolution data needs to be able to solve network power flow problem, and then perform post-solution analysis, e.g. assessment of voltage rise, reverse power flow, feeder power loss etc. within 1 minute interval so that the tool is ready to accept the next sample of 1-min resolution data. The current-mismatch variant of NewtonRaphson algorithm [9] will be deployed in the proposed approach as it incurs less computational efforts.

LV distribution networks are typically of three phase configuration, and more often contains substantial amount of unbalance due to single phase and two phase laterals, load unbalance, and in the recent days due to PV (allocation) related unbalance. Therefore, a three phase network analysis method [10] will be adopted for the proposed approach that can comprehensively model the LV distribution networks with their true complexities including the 3-phase 4-wire configuration, single phase and two phase laterals, neutral grounding, single phase and three phase loads and PV units.

\section{F. Online Quantification of Impacts through Indices}

Each of the solar PV units installed in an LV feeder contributes to the change in network behaviour depending on its capacity. However, to analyse the PV impacts on an overall feeder, a development of numerical indices considering the impacts of all the PV units in a feeder is necessary. The level of PV impact from feeder to feeder will vary depending on the impedance, loading, and level of PV penetration. The use of the numerical indices would also be beneficial to analyse and compare the impacts on different feeders in a distribution system. Indices developed to quantify solar PV impacts in terms of voltage deviation, feeder power flow, MV/LV substation capacity and feeder power loss will be used in the proposed approach. The following proposed indices will be used to investigate the PV impacts using the measured data and also using the results from the load flow analysis for any intended scenario analysis considering changes in the network, such as, increase in PV penetration.

Voltage rise is one of the most discussed impacts of solar PV integration. It can affect the voltage quality of the network by creating a long-duration overvoltage problem [11]. The maximum amount of voltage rise observed among different nodes of a feeder at any given time is used to estimate voltage deviation in the feeder and is defined as the Maximum Voltage Deviation Index (MVDI). At the $k^{\text {th }}$ time instant, this can be obtained using,

$$
\operatorname{MVDI}(k)=\frac{V^{\text {nom }}-V_{\text {max }}(k)}{V^{\text {nom }}}
$$

Here, $V^{\text {nom }}$ is the nominal voltage which can either be a constant, or vary according to the load condition or time of the day, based on the operational policy of the utility; $V_{\max }(k)$ is the voltage at the LV feeder node where the maximum amount of voltage variation is observed at the $k$-th time instant. It is to be noted that MVDI needs to be determined for all the phases separately. The MVDI is an index calculated at any particular instant of time to assess the maximum voltage rise impact of the rooftop solar PV integration in the low voltage network, as given in (2). The index is based on the nominal value of the feeder voltage and therefore, it can provide an indication of the deviation of feeder voltage affected by the level of PV penetration. The index can have both positive and negative value. Without PV integration, the voltages at different points in the feeder are generally lower than the nominal voltage and hence the MVDI will be generally positive. A negative value indicates that the solar PV integration may have produced a voltage-rise. The index can also be used to determine whether the feeder is experiencing overvoltage or undervoltage, by comparing the index with the specified limits for the distribution feeder. For example in Australia, the upper limit is $10 \%$ over the nominal value. An MVDI value which is more negative than -0.1 indicates overvoltage has occurred. Similarly, undervoltage can be indicated by comparing the MVDI with the minimum limit.

An Average Feeder Loading Index (AFLI) of an LV feeder can be formulated as the weighted average of loading levels of all the feeder segments with respect to their lengths. The AFLI at $k^{\text {th }}$ instance can be expressed as,

$$
\operatorname{AFLI}(k)=\left|\sum_{j=1}^{s} \frac{l_{j}}{L_{f}} \times \frac{S_{j}(k)}{C_{j}}\right|
$$

For an $\mathrm{LV}$ feeder, the length of the $j^{\text {th }}$ segment $l_{j}$, total feeder length $L_{f}$ and load carrying capacity of the $j^{\text {th }}$ segment $C_{j}$ are constants. The AFLI will, therefore, depend only on the complex power flow through all the line segments at the $k^{\text {th }}$ time instant, $S_{j}(k)$, where $j=1,2 \ldots, s$ and $s$ is the total number of segments in the feeder. The AFLI index provides an average indication of the degree of loading of a given feeder. It indicates the loading of a distribution feeder as a whole, and not for any particular segment. This index can be used to compare the impacts on the loading of a given feeder for different scenarios of PV generation. The AFLI value is zero if no power flow takes place in the feeder segments. The AFLI value will increase with an increase in feeder power flow to serve the customers. However, with a high penetration of PV resources, AFLI can also increase due to a high amount of reverse power flow.

The amount of the load demand locally served by solar PV units contributes to the release of $\mathrm{MV} / \mathrm{LV}$ substation capacity of an LV feeder. An index is defined to assess the PV impact on the available capacity of substations using a Substation Reserve Capacity Index (SRCI) as given below.

$$
\operatorname{SRCI}(k)=1-\frac{\left|S_{1}^{a}(k)+S_{1}^{b}(k)+S_{1}^{c}(k)\right|}{S_{\text {sub }}}
$$

Here, $S_{1}{ }^{a}, S_{1}{ }^{b}$, and $S_{1}{ }^{c}$ are the complex power flows from phase $a, b$ and $c$ of the LV substation bus and $S_{\text {sub }}$ is the rated capacity of the substation. A value of SRCI equal to one means substation capacity is fully available whereas a value equal to zero means no substation capacity is left unused. With PV units serving local loads, SRCI will decrease with PV integration. However, above a certain level of PV penetration, reverse power flow will cause a higher level of substation capacity to be used and SRCI will decrease.

Less import of power from upstream network due to the 
availability of local PV generation reduces power loss in the distribution feeder. To quantify this, an index is developed using the ratio of the total loss incurred in the feeder to the total load served by the feeder and is referred to as the Feeder Loss to Load Ratio (FLLR), as shown below.

$$
\operatorname{FLLR}(k)=\frac{P_{\text {loss }}^{a}(k)+P_{\text {loss }}^{b}(k)+P_{\text {loss }}^{c}(k)}{P_{\text {dem }}^{a}(k)+P_{\text {dem }}^{b}(k)+P_{\text {dem }}^{c}(k)}
$$

Here, $P_{\text {loss }}{ }^{a}, P_{\text {loss }}^{b}$ and $P_{\text {loss }}{ }^{c}$ are power losses incurred and $P_{d e m}{ }^{a}, P_{d e m}{ }^{b}$ and $P_{d e m}{ }^{c}$ are the load demands in phase a, b and c of an LV feeder. FLLR is an index obtained from the ratio of the loss in the feeder to the load demand of the feeder. Its value increases significantly during midday when the PV output is the highest and the load demand is usually low. During that period, the power loss through the feeder is high due to a high amount of reverse power flow, but since the load demand is typically lower, therefore, the ratio of the loss to the load demand becomes significantly high.

Indices of the Moving Averages (MA) of the variables associated with the PV impact in the sliding windows is required to understand the overall trend of the changes in network behavior caused by solar PV.

The moving average of the voltage deviation index at any given sliding window, $w$, with a width of $W$ can be defined as,

$$
\operatorname{MVDI}_{w}=\frac{\sum_{k=w}^{w+W-1} \operatorname{MVDI}(k)}{W}
$$

Similarly, the moving averages of the other impact indices, $\mathrm{AFLI}_{\mathrm{w}}, \mathrm{SRCI}_{\mathrm{w}}$ and $\mathrm{FLLR}_{\mathrm{w}}$ will be obtained, as given in (7)(9). The moving average provides a useful indicator of the long term trend of the variables associated with the PV impact.

$$
\begin{aligned}
\operatorname{AFLI}_{w} & =\frac{\sum_{k=w}^{w+W-1} \operatorname{AFLI}(k)}{W} \\
\operatorname{SRCI}_{w} & =\frac{\sum_{k=w}^{w+W-1} \operatorname{SRCI}(k)}{W} \\
\operatorname{FLLR}_{w} & =\frac{\sum_{k=w}^{w+W-1} \operatorname{FLLR}(k)}{W}
\end{aligned}
$$

\section{G. Simplified Flowchart of the Proposed On-line Approach}

Fig. 4 shows a simplified flow chart of the major functions of the proposed approach. The width of the sliding window, $W$ is taken as input from the user. Each of the sliding window, specified by the window counter, $w$, contains $W$ number of 1 min assessment outcomes. The inner loop, consisting of online data collection, pre-processing, load flow analysis (if required for "what-if" analysis), PV impact assessment through quantification indices, and storing of 1-min results, continues until $k$ becomes equal to $W$. Once the assessment for the $w^{\text {th }}$ sliding window is obtained, results are displayed from $w^{\text {th }}$ to $(w+W-1)^{\text {th }}$ number of time instances. A summary of the assessment, such as maximum value, minimum value, moving average value etc. for the latest sliding window can be made available for reporting.

\section{ApPLiCATION in REAL TIME EnVIRONMENT}

With the availability of the real time distribution network model compiled from the most updated information, the proposed approach can be used for determining the network behavior under any probable changes in PV output, load demand, or even changes in network topology resulting from outage or switching operation. With the capability to assess the solar PV impacts on distribution networks on-line, the proposed approach can also be used for monitoring of dynamic events such as fluctuations induced by passing clouds, etc. Further, this approach may also aid in the decision making for compensating the adverse impacts of these dynamic events by proving online assessment of the perturbed parameters. A brief description of these capabilities is provided below.

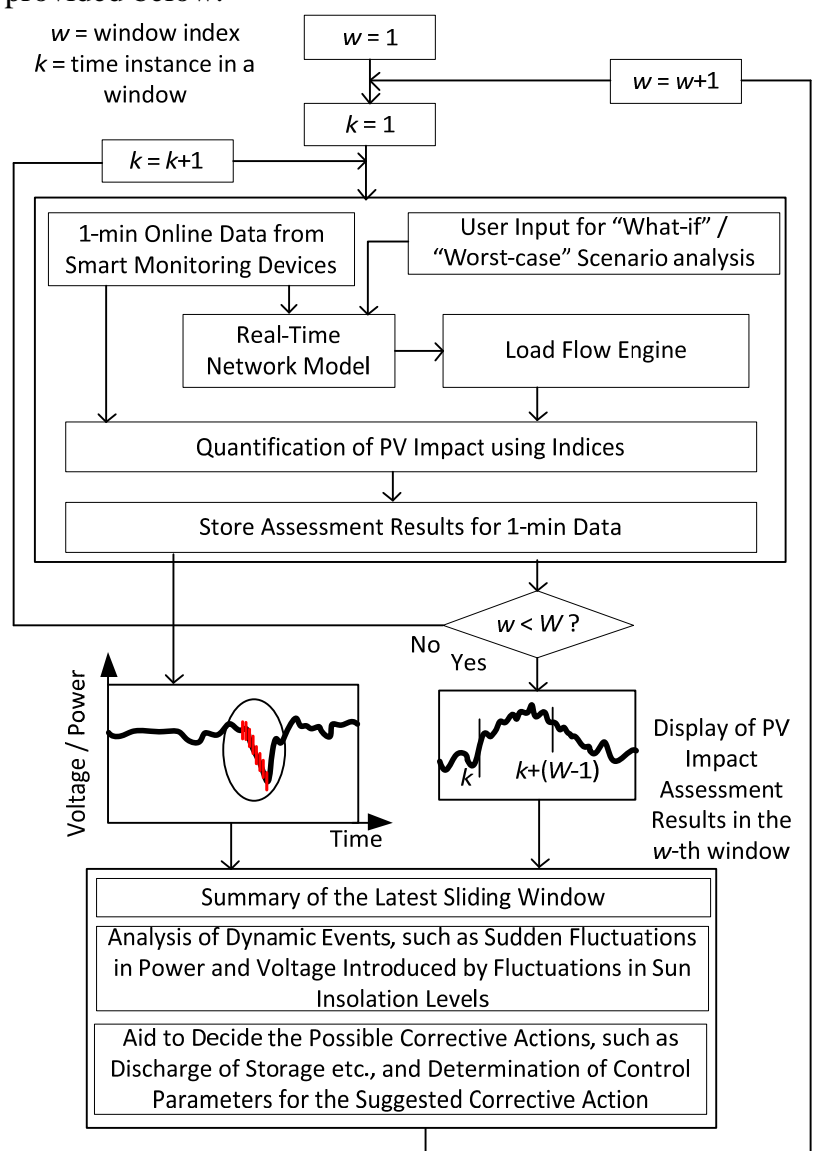

Fig. 4. A simplified flowchart of the proposed on-line assessment approach.

\section{A. Dynamic "What-if" Analysis}

With an increasing level of solar PV penetration, network operators often need to investigate how the networks would operate under a changed condition of load and PV output. This type of investigation is typically performed using "what-if" type of analysis by first selecting a base case scenario, and then by varying the parameter of interest (for example, the PV output, consumer load, etc). Conventionally, a base case scenario would typically consist of historical off-line data or synthetic data, and therefore, may not always represent the actual threats of high PV penetration in an operating distribution network. In a real-world scenario, network 
condition changes with the variations in load demands, PV outputs and network configurations caused by switching operation. Impacts of high PV penetration would be different under different network conditions. Therefore, a static base case using off-line data may not be sufficient to assess high PV penetration impacts. Rather a dynamic base case changing with time and reflecting the actual changes in the network would be needed. The real-time distribution network model obtained using the proposed approach can be used as base case for such a dynamic "what-if” analysis.

A simplified block diagram of the dynamic "what-if" analysis is shown in Fig. 5. The proposed online approach compiles time-variant base case scenarios using the real-time load, PV and network data obtained from the actual distribution network using the Advanced Metering Infrastructure. A base case scenario at the $k$-th time instant will be constructed using the active and reactive load demand, $P_{\mathrm{L}}(k)$ and $Q_{\mathrm{L}}(k)$, the PV output at $P_{\mathrm{PV}}(k)$, and the network configuration, each at the $k$-th time instant. The changed network configuration will affect the network admittance matrix $Y(k)$. The user, e.g., a network operator, would be able to modify the compiled base case scenario to investigate a potential PV penetration scenario to assess the impacts, or, possibility of any threat on the network under that potential scenario.

Once the "what-if" analysis for the $k$-th instant is finished using the load flow engine, results are reported to the operator and the system is ready for analysis for the $(k+1)$-th instant.

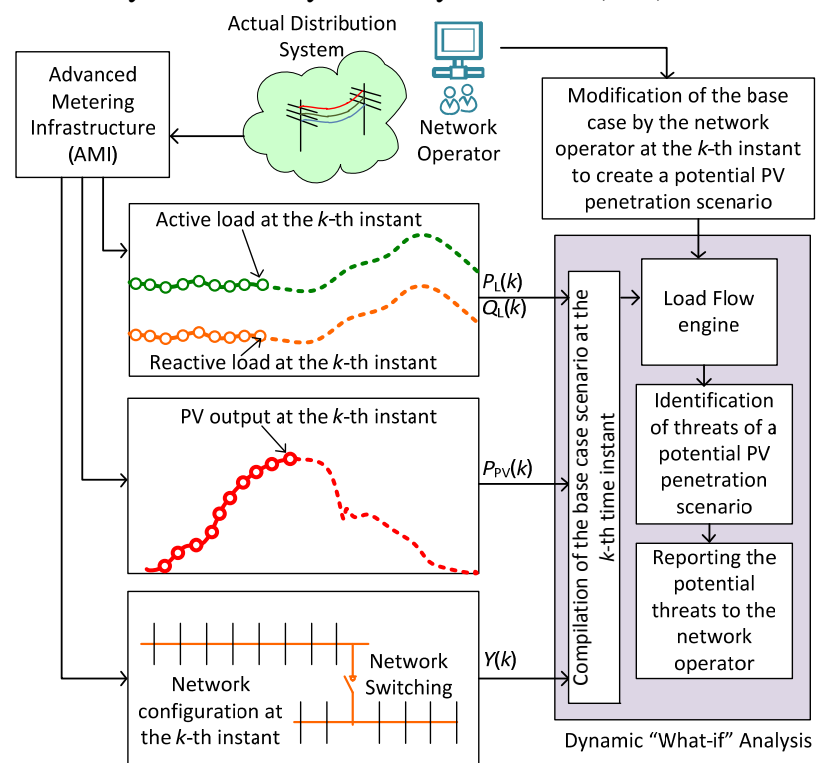

Fig. 5. Dynamic "what-if" analysis

\section{B. Online Monitoring of PV Output Fluctuations}

The power output of PV resources depends on the incident sun insolation on PV panels. The PV impacts, therefore, changes throughout the day in accordance with the sun insolation. Any event that creates a sudden change in the sun insolation level, such as passing clouds over the PV installation site [12], eventually produces fluctuations in the $\mathrm{PV}$ output and this is reflected in the feeder voltage and power flow. From the collected real-time data, the proposed approach will be able to provide an online assessment of the fluctuating behavior of network caused by sudden and irregularly variable PV outputs. The ramp rate of the PV output changes during cloud passing can be very high [11] and this will affect the ramp rate of the resulting voltage variation.

Indices are defined to quantify the ramp rate of PV power output variation caused by cloud passing and the resulting voltage variation. A sudden change of the total amount of the PV output in the feeder is considered as a potential impact of PV output fluctuation, which is expressed using Instantaneous Power Ramp Rate (IPRR) with a unit of $\mathrm{kW}$ per unit of time, as shown below.

$$
\operatorname{IPRR}(k)=\frac{\sum_{i=1}^{n} P_{i}^{P V}(k)-\sum_{i=1}^{n} P_{i}^{P V}(k-1)}{t(k)-t(k-1)}
$$

where, $P_{i}^{P V}(k)$ is the PV power at the $i$-th node of the feeder at $k$-th time instant; $i=1,2, \ldots, n$, where, $n$ is the total number of nodes in the feeder. Positive IPRR indicates an increase of feeder PV generation and negative IPRR indicates a decrease of PV generation. The maximum amount of voltage variation observed in a feeder is used for evaluating the voltage ramp rate. The proposed index is defined as Instantaneous Voltage Ramp Rate (IVRR) that indicates the amount of voltage change between the present and the immediate past instant of measurement. The IVRR is expressed in terms of Volts per unit of time and is calculated using the following equation for the $k^{\text {th }}$ time instant.

$$
\operatorname{IVRR}(k)=\frac{V_{\max }(k)-V_{\text {max }}(k-1)}{t(k)-t(k-1)}
$$

In (11), $V_{\max }(k)$ is the voltage at the LV feeder node where the maximum amount of voltage variation is observed and $t(k)$ is the time corresponding to $k^{\text {th }}$ time instant. The sign of IVRR indicates the direction of voltage ramp; positive sign means an increase in voltage as compared to previous measurement and a negative sign indicates a decrease in voltage. High resolution time series data (in the scale of seconds) would be more suitable for the measurements of IPRR and IVRR. The same mathematical formulations of IPRR and IVRR given in (10) and (11), respectively, will be applicable when such high resolution data is available. The moving averages of IPRR and IVRR can be calculated in the same way as shown in (6)-(9) to obtain long term trends of the fluctuations.

\section{Aiding the Decision Making for PV Impact Mitigation}

Online monitoring and measurements of PV impacts can be used for real time decision making for mitigation of PV impacts. For example, if any immediate action is to be taken for mitigating the voltage rise caused by PV, the amounts and the exact time references of occurrence of the voltage rise have to be provided to the controllers of the compensating devices; an on-line impact assessment approach would be helpful in this case. Similarly, if storage devices are used for smoothing out the PV fluctuations [13] caused by passing clouds, the voltage and power ramp rates available from online measurements can be used for determining the charge and 
discharge control parameters of storage devices.

A generic conceptual representation of assisting the PV impact mitigation by the proposed approach is shown in Fig. 6(a) using the rate of change of a fictitious Fluctuating Variable (FV). The detection of a sudden fluctuation can be performed from the abrupt change in the ramp rate of the FV. A Compensating Action (CA) designed to mitigate the fluctuating effect has to be triggered at the point of detection. The ramp rate of the compensating action will depend on the desired ramp rate of the FV, which is expressed by (12) for the $k$-th time instant.

$$
\left.\frac{\Delta(\mathrm{CA})}{\Delta t}\right|_{k}=\left.\frac{\Delta(\mathrm{FV})}{\Delta t}\right|_{k} ^{\text {desired }}-\left.\frac{\Delta(\mathrm{FV})}{\Delta t}\right|_{k} ^{\text {actual }}
$$

The ramp rate of the compensating action has to be changed according to the ramp rate of the fluctuating variable at each instant of time. For example, to mitigate the impacts of PV output fluctuation, a compensating action can be implemented using a Community Energy Storage (CES) [14] that may be used for smoothing out the fluctuations in PV generation in the feeder, as shown in Fig. 6(b). The ramp rate of the storage discharge power needs to be adjusted using (12) based on the IPRR value obtained by (10). The discharge ramp rate has to be adjusted at each instant of time based on the most recent IPRR value. Based on the discharge power ramp rate, the discharge current ramp rate of the storage device can be determined at each instant of time and can be sent to the storage controller for implementation. In this way, the proposed approach can aid in the decision making for a real time mitigation of PV impacts. While the proposed assessment approach could be used to assist in the decision making for mitigation of an ongoing PV impact in real time, it would not in itself be able to mitigate the impact, or control the action of mitigation.
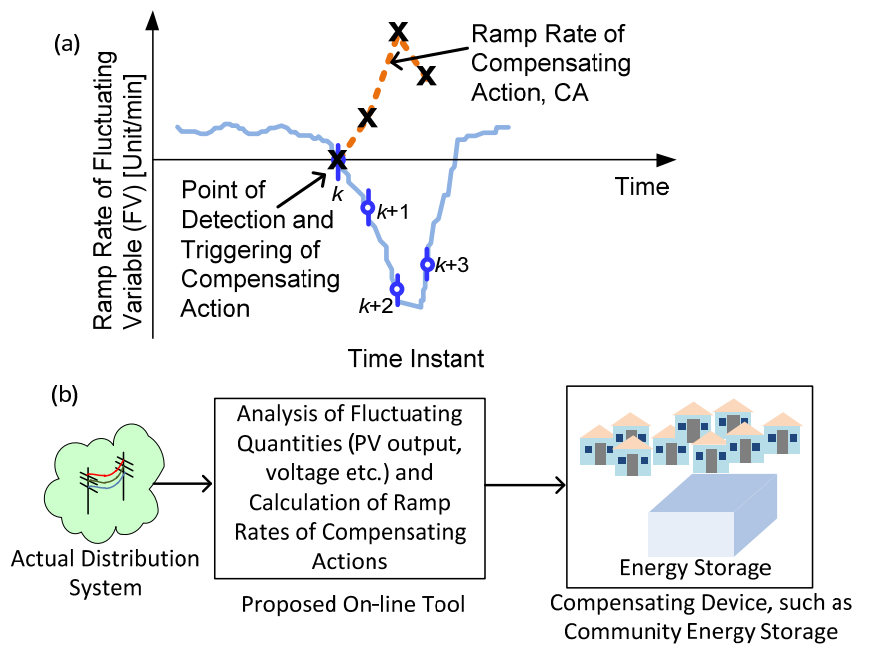

Fig. 6. Application of the proposed approach for mitigation of the impact of PV output fluctuations: (a) calculation of ramp rate of a generic compensating action; (b) translation of the idea in terms of a community storage device.

\section{EXAMPLE APPLICATIONS}

The proposed on-line assessment approach is applied on a typical LV distribution feeder in Australia that is connected to a real $11 \mathrm{kV}$ distribution network derived from the distribution system in the South-East part of New South Wales. It is an 80 $\mathrm{km}$ long rural system with 3 voltage regulators. The MV network and LV feeder diagrams are shown in Fig. 7. The LV feeder is connected to bus 68 of the $11 \mathrm{kV}$ network, as marked using an arrow and a rectangular box in Fig. 7. It contains households with rooftop PV units. Load profiles captured from a residential LV distribution system in Australia is used to develop voltage dependent household load models. Voltage dependency parameters of typical electric appliances [15] found in residential households were used for an aggregate modeling of the household loads. PV systems, each rated at 4 $\mathrm{kW}$, are integrated with the feeder at different customer premises. The PV inverters are operated at unity power factor. The data of the test feeder is provided in Table I. Though this paper shows results corresponding to one of the LV feeders, it can be similarly applied for other LV feeders in the network.

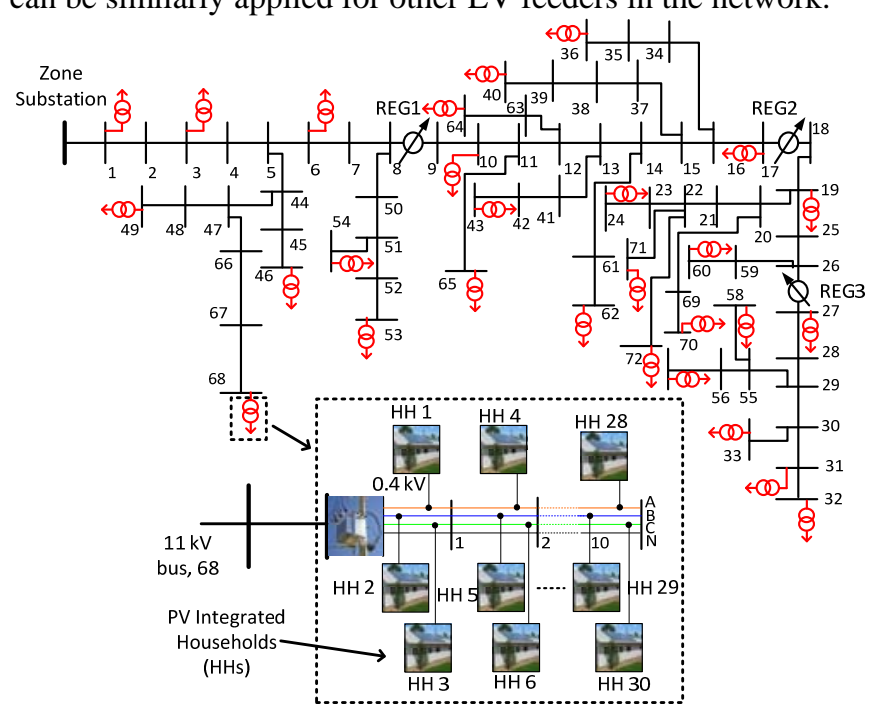

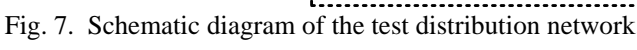

\begin{tabular}{|c|c|} 
TABLE I: TEST LV FEEDER DATA \\
\begin{tabular}{|c|c|}
\hline Feeder Length (metre) & 350 \\
\hline Number of Households & 30 \\
\hline Pole to Pole Distance (metre) & $30-40$ \\
\hline Conductor & $7 / 3.00 \mathrm{AAC}$ \\
\hline MV/LV Transformer Size & $160 \mathrm{kVA}$ \\
\hline PV Module / Inverter & Kyocera / SMA Sunnyboy \\
\hline
\end{tabular}
\end{tabular}

\section{A. Online Monitoring of PV Impacts in Terms of Indices}

The indices developed are used to monitor the PV impacts. Fig. 8 shows the voltage deviation impact caused by the solar PV on the test feeder in terms of MVDI. The MVDI becomes negative due to the voltage rise caused by PV after 9 AM, as shown in Fig. 8(a), and continues to increase in the negative direction. A switch-over to 15-min sliding windows at this time can provide a larger view of the changes in voltage that takes place in the feeder due to the PV penetration. This is shown in Fig. 8(b). The MVDIs for all phases reduce to their peak negative levels at about 1 PM, as shown in Fig. 8(c). The numerical values of MVDIs indicate that voltage rise is approaching the overvoltage limit of $10 \%$, as specified by Australian standards. Based on the severity of the situation, a 5-min sliding window is chosen for detailed analysis, as 
shown in Fig. 8(d).

Although the width of the assessment window is selected at the user's discretion, a feature can be developed in the implementation of the proposed approach to make a suggestion to the operator to narrow down the width with the increase of the irradiance level. This would be useful to pay attention to the voltage rise impact before it reaches a limit violation level.

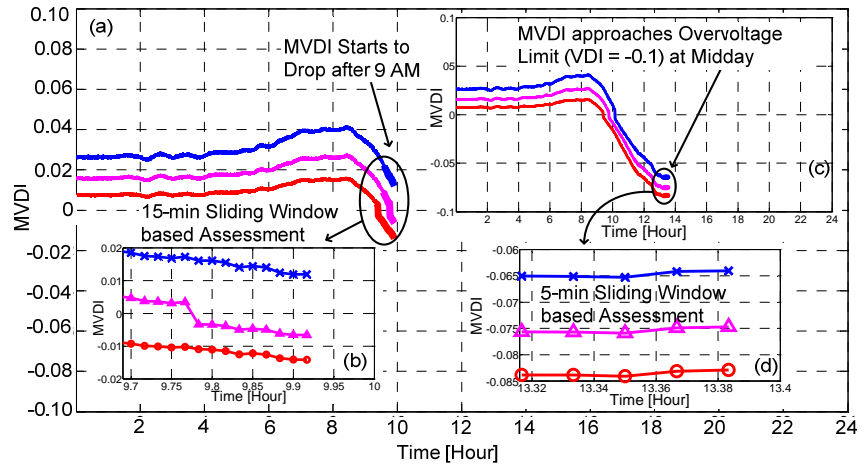

Fig. 8. On-line monitoring of voltage deviation caused by PV: (a) Voltage starts to rise above nominal value as indicated by negative MVDI; (b) A 15min sliding window of the situation in Fig. 8(a); (c) MVDI approaches overvoltage limit; (d) A 5-min assessment window to obtain a closer view of the situation in Fig. 8(c).

As the loads at the PV connection points are now served locally, less power is imported from the upstream network over the feeder and hence, the feeder loading is reduced. However, with the increase of the PV output power as the day progresses, the loading may increase due to the reverse power flow caused by the surplus power from PV units. Illustrations of these events are presented in Fig. 9, where in Fig, 9(a) it is shown that the feeder loading, expressed in terms of AFLI, starts to drop at about 9 AM when the PV units starts to generate sufficient amount of power to share a significant portion of the loads. A 15-min assessment window corresponding to this time is shown in Fig. 9(b) that provides a more precise view of the situation.

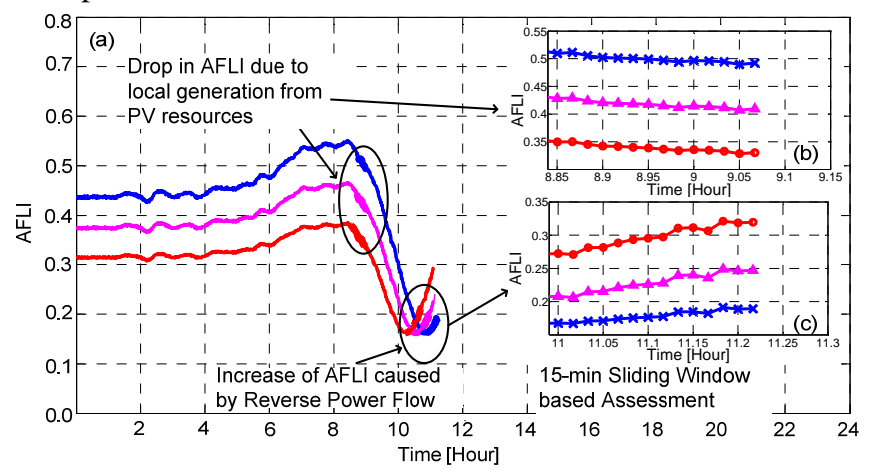

Fig. 9. On-line monitoring of feeder loading variation caused by PV: (a) Decrease of AFLI caused by PV units serving local loads and increase of AFLI in the midday caused by reverse power flow; (b) a 15-min sliding window of the decrease in AFLI; (c) a 15-min sliding window of the increase in AFLI

With enough PV output power to exceed the load demand at the connection points, the AFLI values starts to increase at about 11 AM as shown in Fig. 9(a) and a detailed view of this is shown in Fig. 9(c) using another 15-min assessment window.

Similar effect is observed with substation loading, shown in
Fig. 10 with the index of the remaining capacity of substation, SRCI. The increase in SRCI starting at about $9 \mathrm{AM}$, as shown in Fig. 10(a), indicates the release of substation capacity due to the fact that the PV units are contributing to serve the local loads at the PV connection points. This is followed by a dropping trend from about $11 \mathrm{AM}$ due to the loading of the substation caused by the reverse power flow into the upstream network, also shown in Fig. 10(a). Both of the increasing and decreasing trends or SRCI are more accurately assessed using 15-min sliding windows, as shown in Fig. 10(b) and 10(c), respectively.

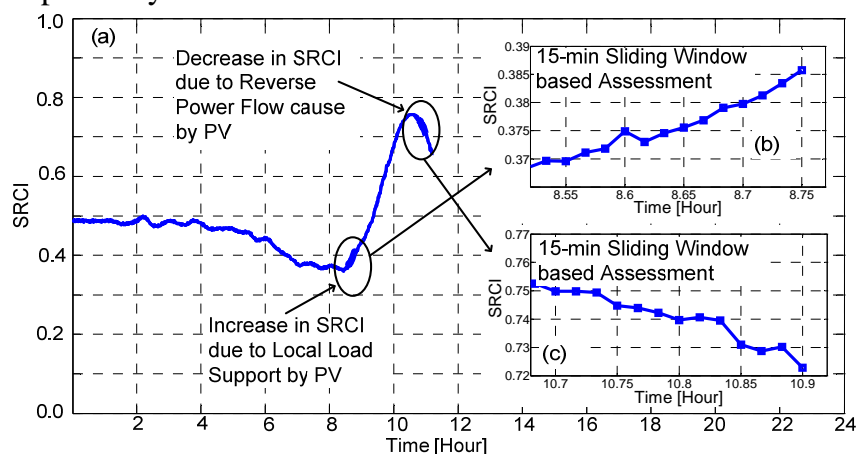

Fig. 10. On-line monitoring of substation loading variations caused by PV: (a) Increase of SRCI caused by PV units serving local loads and decrease of SRCI in the midday caused by reverse power flow; (b) a 15-min sliding window of the increase in SRCI; (c) a 15-min sliding window of the decrease in SRCI.

The power loss in the feeder decreases with the reduction in power import from the upstream network with the availability of PV power, which is shown in Fig. 11(a) with the drop in FLLR at $9 \mathrm{AM}$ in the morning. However, when the surplus power flows through the feeder towards the upstream substation, the power loss in the overall feeder increases, which is shown in Fig. 11(a), with the increase in FLLR, at about 11 AM. The increasing and decreasing trends of FLLR are assessed using the 15-min sliding windows and shown in Fig. 11(b) and 11(c).

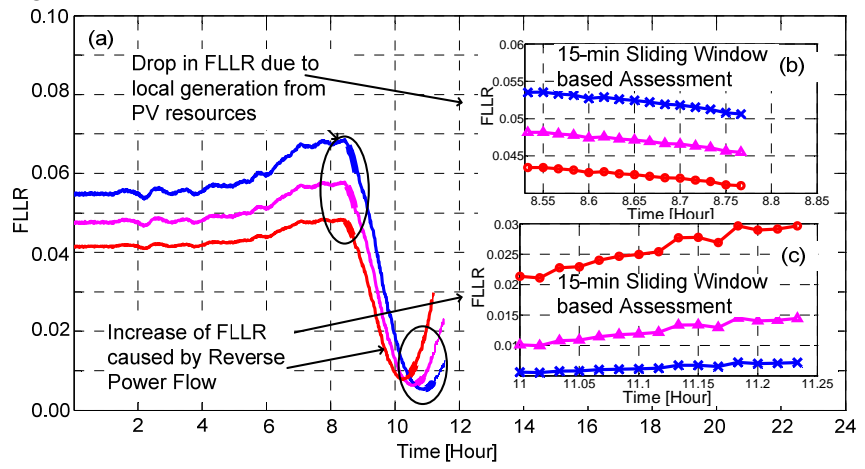

Fig. 11. On-line monitoring of feeder power loss variation caused by PV: (a) Decrease of FLLR caused by PV units serving local loads and increase of FLLR in the midday caused by reverse power flow; (b) a 15-min sliding window of the decrease in FLLR; (c) a 15-min sliding window of the increase in FLLR.

A summary report of the indices for the latest sliding window is available for an overview of the PV impacts as shown in Fig. 12. Once the full 24-hour period is completed, the trends of the impact indices and assessment reports over the entire day are available, as shown in Fig. 13. Variations in feeder voltage caused by PV is shown in terms of MVDI in 
Fig. 13(a), variations in feeder loading is shown in Fig. 13(b) in terms of AFLI, variation of the usage of substation capacity is shown in Fig. 13(c) using SRCI, and variations in feeder power loss is shown in Fig. 13(d) using FLLR.

\begin{tabular}{|c|c|c|c|c|c|}
\hline \multicolumn{6}{|c|}{ Summary of the Latest Sliding Window } \\
\hline \multicolumn{4}{|l|}{ Width of Sliding Window } & 15 & $\min$ \\
\hline Impact Indices & Min & Max & Moving & & \\
\hline MVDI & -0.063 & -0.066 & -0.06 & & \\
\hline AFLI & 0.476 & 0.497 & 0.48 & & \\
\hline SRCI & 0.220 & 0.247 & 0.23 & & \\
\hline FLLR & 0.045 & 0.050 & 0.04 & & \\
\hline \multicolumn{4}{|c|}{ Violation of Overvoltage / Under Voltage Limit } & No & Yes/No \\
\hline \multicolumn{4}{|l|}{ Detection of Sudden Fluctuation } & No & Yes/No \\
\hline \multicolumn{4}{|c|}{ Maximum Voltage Change betn Two Consecutive Measurements } & 0.424 & Volts \\
\hline \multicolumn{4}{|c|}{ Maximum Power Change betn Two Consecutive Measurements } & 1.61 & kW \\
\hline
\end{tabular}

Fig. 12. A summary report of PV impacts in the latest sliding window
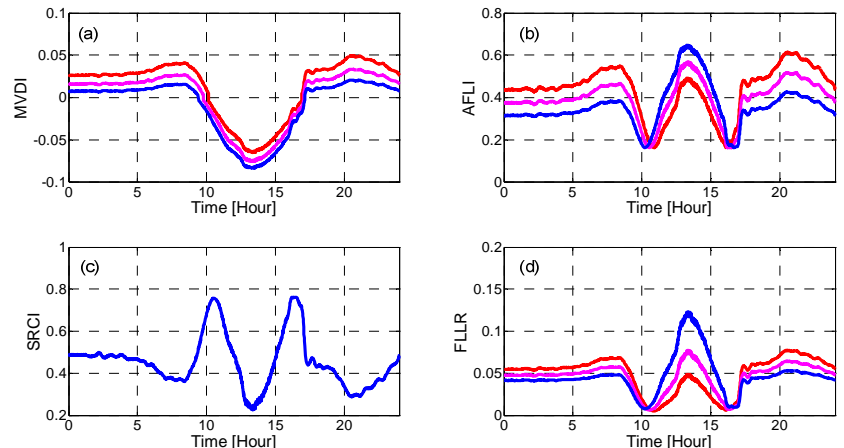

Fig. 13. PV impact indices after completion of a 24-hour period: (a) MVDI; (b) AFLI; (c) SRCI; (d) FLLR.

\section{B. Applications of Dynamic "What-if" Analysis}

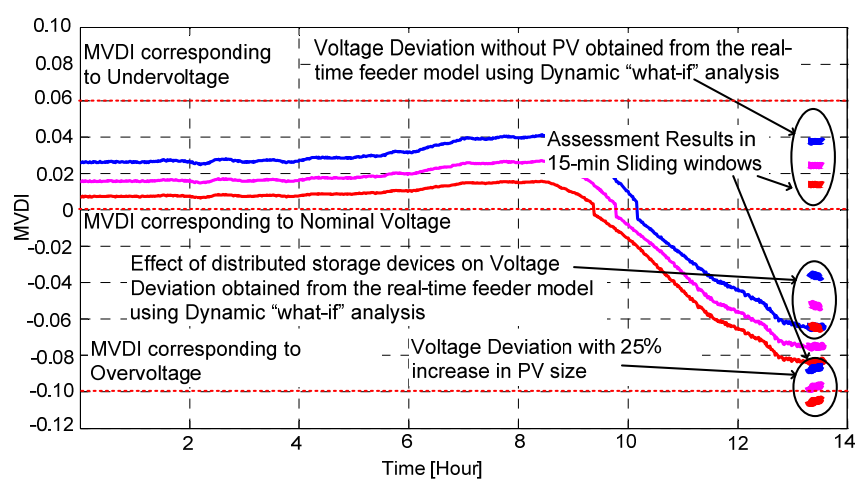

Fig. 14. Application of dynamic "what-if” analysis using MVDI.

A simple, yet attractive application of the dynamic "whatif" analysis is to investigate how the network would behave without PV penetration to understand how the integration of PV has affected the system operation. For example, an operator might be interested to find out what would be the voltage deviation at midday without PV. This information can be obtained by performing a "what-if" analysis by forcing the PV outputs to zero on the real-time network model at midday. The proposed approach was used to perform such an analysis and the results are shown in terms of voltage deviation index in Fig. 14. It is shown that without PV, the MVDIs for different phases are within 0.01 to 0.04 at midday which indicates the voltage would be lower than nominal, but within the normal operating range.
Another "what-if" analysis can be performed to investigate the impact of increasing PV generation on real-time basis at about 1 PM. The sizes of solar PV units at different feeder nodes were increased by $25 \%$ of the present size. The "whatif" analysis indicates a violation of the overvoltage limit with this increase in PV size as shown in Fig 14.

A network operator can also use the "what-if" analysis feature to investigate the effectiveness of a potential mitigation strategy. For example, the impact of using distributed storage devices as a voltage rise mitigation strategy can be analyzed by incorporating storage device models with the real-time distribution feeder model. Results of such a "what-if" analysis are shown in Fig. 14, where storage devices are used in the midday to consume surplus PV power when MVDI values reach nearly at the limit violation level. Distributed storage devices are operated in this case to consume surplus PV power and as a result voltage rise is partially mitigated, as observed from the MVDI values.

\section{Online Impact Assessment of PV Output Fluctuations}

The impact indices proposed in this paper are applied to assess the impacts of PV output fluctuations. A practical solar PV output profile obtained from the Renewable Energy Integration Facility at CSIRO Energy Technology in Australia was used for the example application. It is observed in the PV output data that the PV output was distorted by fluctuations in the sun irradiance level.

The impacts of PV output fluctuations on the feeder are shown in Fig. 15 in terms of the proposed impact indices. The actual PV generation in kilowatts is shown in Fig. 15(a) that shows the PV output fluctuates several times in the period from 9:00 hours to 13:00 hours. The PV output suddenly reduces to less than $5 \mathrm{~kW}$ from about $107 \mathrm{~kW}$ at about 12:50 PM. With respect to the total rated PV capacity, this fluctuation is more than $90 \%$, as shown in Fig. 15(b). The IVRR caused by such reduction in PV output is shown in Fig. 15 (c) which shows the voltage reduces by $10 \%$.
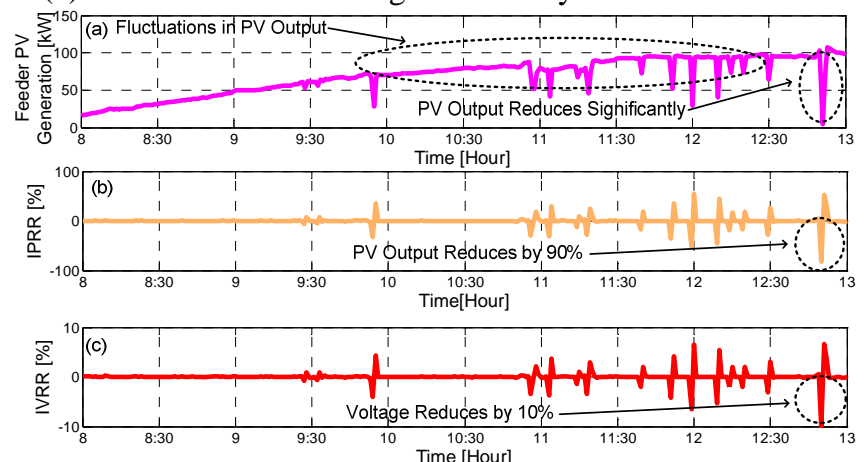

Fig. 15. Fluctuations in PV output (a) Fluctuations in total PV generation in the feeder; (b) IPRR in percent of total PV capacity in the feeder; (c) IVRR in percent of the feeder nominal voltage.

A 60 -min sliding window, from 12 hours to 13 hours, is used to observe the consecutive fluctuations in a single window, as shown in Fig. 16. The fluctuations in MVDI are shown in Fig. 16(a) at the time instances corresponding to the PV output fluctuations in Fig. 15(a). Similarly, Fig. 16(b), 16(c), and 16(d) show the fluctuations in AFLI, SRCI and FLLR at the same time instances as in MVDI. 
In summary, the proposed online approach would be useful for the distribution network operators to observe the impacts of the present level of PV penetration on their networks in a real time environment. Using the dynamic "what-if" analysis feature of this approach, the network operators would also be able to understand the impacts of a potential PV penetration level on the present or actual condition of their network. Also, the impacts of ongoing PV output fluctuations on network voltage can be assessed using the proposed online approach as demonstrated through simulation results.
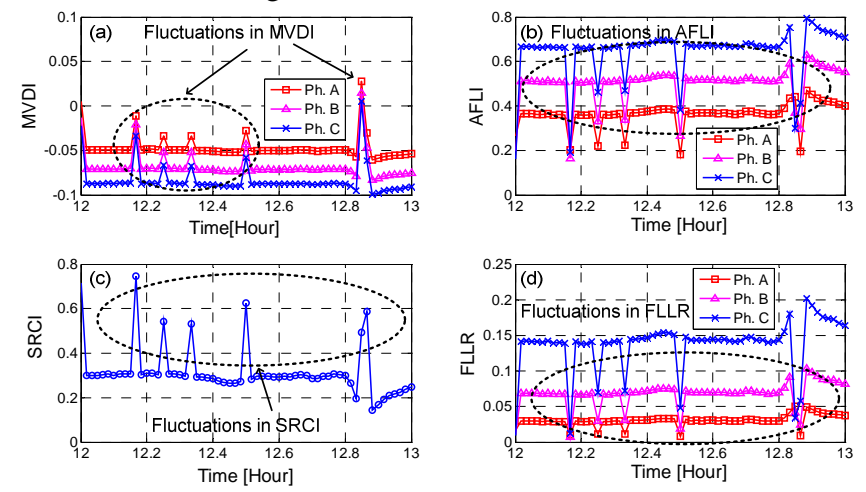

Fig. 16. Online assessment PV output fluctuation with impact indices in a 60min sliding window: (a) MVDI; (b) AFLI; (c) SRCI; (d) FLLR.

\section{CONCLUSION}

A variable-width sliding window based on-line approach has been proposed for investigation of rooftop solar PV impacts on LV distribution networks. The proposed approach will take on-line network data as input and then will analyze solar PV impacts on the network using a set of proposed impact indices. Using the variable-width sliding window allows the width of the assessment window to be varied depending on the situation. A narrow window can be used during peak PV period, while a wider window can be used during low or no PV output period. This can help to avoid unnecessary monitoring activities. With the availability of most updated network topology, load and PV output data, this approach can make use of real-time models of distribution networks for dynamic "what-if” analysis that would be useful for the assessment of solar PV impacts under a given PV generation scenario using the actual network condition at a given instant of time. A summary report of the assessment outcomes is also available for an 'at-a-glance' view of the impacts over a given period. The information obtained using the proposed approach would be beneficial to implement mitigation actions against adverse impacts of PV penetration, including fluctuations caused by sudden variations in PV output.

\section{ACKNOWLEDGMENT}

The authors gratefully acknowledge the support and cooperation of Essential Energy, Endeavour Energy and CSIRO Energy Technology for providing practical system information and data for this research.

\section{REFERENCES}

[1] B. H. Chowdhury and A. W. Sawab, "Evaluating the value of distributed photovoltaic generations in radial distribution systems," IEEE Transactions on Energy Conversion, vol. 11, pp. 595-600, 1996.

[2] R. A. Walling, R. Saint, R. C. Dugan, J. Burke, and L. A. Kojovic, "Summary of Distributed Resources Impact on Power Delivery Systems," IEEE Transactions on Power Delivery vol. 23, pp. 1636-1644, 2008.

[3] V. H. M. Quezada, J. R. Abbad, and T. G. S. Roman, "Assessment of energy distribution losses for increasing penetration of distributed generation," IEEE Transactions on Power Systems, vol. 21, pp. 533-540, 2006.

[4] J. A. Martinez and J. Mahseredjian, "Load flow calculations in distribution systems with distributed resources. A review," in IEEE PES General Meeting, Detroit, Michigan, USA, 24-29 July, 2011, pp. 1-8.

[5] L. F. Ochoa, A. Padilha-Feltrin, and G. P. Harrison, "Evaluating distributed generation impacts with a multiobjective index," IEEE Transactions on Power Delivery, vol. 21, pp. 1452-1458, 2006.

[6] P. Chiradeja and R. Ramakumar, "An approach to quantify the technical benefits of distributed generation," IEEE Transactions on Energy Conversion, vol. 19, pp. 764-773, 2004.

[7] R. C. Dugan and M. McGranaghan, "Sim City," Power and Energy Magazine, IEEE, vol. 9, pp. 74-81, 2011.

[8] S. Zhang, C. Zhang, and Q. Yang, "Data preparation for data mining," Applied Artificial Intelligence, vol. 17, Issue 5-6, pp. 375-381, May-Jul., 2003. Online publication date Nov. 2010.

[9] P. A. N. Garcia, J. L. R. Pereira, S. Carneiro, Jr., V. M. da Costa, and N. Martins, "Three-phase power flow calculations using the current injection method," Power Systems, IEEE Transactions on, vol. 15, pp. 508-514, 2000.

[10]M. J. E. Alam, K. M. Muttaqi, and D. Sutanto, "A Three-Phase Power Flow Approach for Integrated 3-Wire MV and 4-Wire Multigrounded LV Networks With Rooftop Solar PV," IEEE Transactions on Power Systems, vol. 28, pp. 1728-1737, 2013.

[11]"IEEE Recommended Practice for Monitoring Electric Power Quality," IEEE Std 1159-2009 (Revision of IEEE Std 1159-1995), pp. c1-81, 2009.

[12]E. Kern Jr, E. M. Gulachenski, and G. A. Kern, "Cloud effects on distributed photovoltaic generation: Slow transients at the Gardner, Massachusetts photovoltaic experiment," IEEE Transactions on Energy Conversion, vol. 4, pp. 184-190, 1989.

[13]T. D. Hund, S. Gonzalez, and K. Barrett, "Grid-Tied PV system energy smoothing," in 35th IEEE Photovoltaic Specialists Conference (PVSC), Honolulu, HI, USA, 20-25 Jun., 2010, pp. 2762-2766.

[14]R. J. Yinger and A. E. Kamiab, "Good Vibrations," Power and Energy Magazine, IEEE, vol. 9, pp. 22-32, 2011.

[15] K. P. Schneider and J. C. Fuller, "Detailed end use load modeling for distribution system analysis," in Power and Energy Society General Meeting, 2010 IEEE, 2010, pp. 1-7.

\section{BIOGRAPHIES}

M J E Alam (S'10) obtained his B Sc and M Sc degree in EEE from Bangladesh University Engineering and Technology in 2005 and 2009, respectively. At present, he is working towards $\mathrm{PhD}$ degree at the University of Wollongong, New South Wales, Australia. His research interest includes modeling and analysis of power systems considering the impacts of distributed and renewable energy resources. He has worked in the electric utility industry in Bangladesh for 4.5 years

K M Muttaqi (M'01, SM'05) received the Ph.D. degree from Multimedia University, Malaysia, in 2001. Currently, he is an Associate Professor at the School of Electrical, Computer, and Telecommunications Engineering, University of Wollongong, Wollongong, Australia. He is an Associate Editor of IEEE Transactions on Industry Applications. His special fields of interests include distributed generation, renewable energy, power system planning, intelligent grid, and power system reliability.

D Sutanto (SM'89) obtained his BEng. (Hons) and PhD from the University of Western Australia. He is presently the Professor of Power Engineering at the University of Wollongong, Australia. His research interests include power system planning, analysis and harmonics, FACTS and Battery Energy Storage systems. He is a Senior Member of IEEE. He is currently the IEEE IAS Area Chair for Region 10 (Asia Pacific). 